\title{
YET ANOTHER PROOF OF THE LYAPUNOV CONVEXITY THEOREM
}

\author{
ZVI ARTSTEIN
}

(Communicated by R. Daniel Mauldin)

\begin{abstract}
A new proof is given, of the convexity and compactness of the range of an atomless $R^{n}$-valued measure.
\end{abstract}

Several proofs are available for the theorem of A. A. Lyapunov on the range of a vector measure. (The bibliography given here is not exhaustive.) These proofs reflect both the applicability and the value of the theorem. This paper presents yet another proof, one based on a new, useful argument.

The measure theory we use is standard. Let $(\Omega, \Sigma)$ be a measurable space, and let $\mu=\left(\mu_{1}, \ldots, \mu_{n}\right)$ be an atomless $R^{n}$-valued $\sigma$-additive finite measure on it. The range of the restriction of $\mu$ to a set $T$ in $\Sigma$ is

$$
R(T)=\{\mu(A): A \subset T, A \in \Sigma\} .
$$

We denote by $|\mu|$ the scalar measure of total variation of $\mu$. From here on we identify sets which differ by only a set of $|\mu|$-measure zero. Thus $T_{1} \subset T_{2}$ means that $|\mu|\left(T_{1} \backslash T_{2}\right)=0$. We denote by $\operatorname{ch} K$ the closed convex hull of the set $K \subset R^{n}$. With this notation the Lyapunov theorem reads $\operatorname{ch} R(\Omega)=R(\Omega)$. We arrive at it as the conclusion of the following result.

Theorem. Let $x$ be in chR( $\Omega)$. consider the subclass $\Sigma^{1}$ of $\Sigma$, consisting of those $T \in \Sigma$ such that $x \in \operatorname{ch} R(T)$. Then $\Sigma^{1}$ contains a minimal set, say $S$, with respect to inclusion (minimal up to $|\mu|$-null set). For the minimal set $S$ we have $\mu(S)=x$. In particular $x \in R(\Omega)$, and the latter is therefore closed and convex.

We use the following result.

Lemma. Let $T=\bigcap_{i=1}^{\infty} T_{i}$, where $T_{1} \supset T_{2} \supset \cdots$ is a decreasing sequence in $\Sigma$. Then $\operatorname{ch} R(T)=\bigcap_{i=1}^{\infty} \operatorname{ch} R\left(T_{i}\right)$.

Proof. The inclusion of $\operatorname{ch}(R(T))$ in the intersection is trivial. To verify the other direction, and since all the sets are compact, it suffices to prove that if $y_{i} \in \operatorname{ch} R\left(T_{i}\right)$ then the distance between $y_{i}$ and $\operatorname{ch}(R(T))$ tends to zero as

Received by the editors May 24, 1988.

1980 Mathematics Subject Classification (1985 Revision). Primary 28B05. 
$i \rightarrow \infty$. Since the closure and taking convex hull operations do not increase the distance from the convex set $\operatorname{ch} R(T)$, it is enough to verify the claim for $y_{i} \in R\left(T_{i}\right)$, namely when $y_{i}=\mu\left(A_{i}\right)$ for $A_{i} \subset T_{i}$. In particular $y_{i}=\mu\left(A_{i} \cap\right.$ $T)+\mu\left(A_{i} \backslash T\right)$. The first term belongs to $R(T)$; the second term is bounded in norm by $|\mu|\left(T_{i} \backslash T\right)$. The latter sequence converges to zero (an elementary fact of scalar measures, implied by the $\sigma$-additivity); hence the vectors $y_{i}-\mu\left(A_{i} \cap T\right)$ tend to zero and this verifies the claim.

Proof of the existence of a minimal element in $\Sigma^{1}$. Let $T_{\gamma}, \gamma \in \Gamma$, be a decreasing family, not necessarily countable, of sets in $\Sigma^{1}$. We claim that a cofinal subsequence $T_{\gamma_{i}}, i=1,2, \ldots$, exists; namely, each $T_{\gamma}$ contains an element of the sequence. To show this, consider the numbers $|\mu|\left(T_{\gamma}\right), \gamma \in \Gamma$, and choose a sequence $|\mu|\left(T_{\gamma_{i}}\right)$ among these numbers such that each $|\mu|\left(T_{y}\right)$ is greater than or equal to one of the elements in the sequence; $T_{\gamma_{i}}$ is then cofinal. Clearly, $T=\bigcap_{i=1}^{\infty} T_{\gamma_{i}}$ is included (up to $|\mu|$-null sets) in each $T_{\gamma}$. By the lemma, if each $T_{\gamma}$ belongs to $\Sigma^{1}$, then $T \in \Sigma^{1}$; i.e. $T_{\gamma}, \gamma \in \Gamma$, has a lower bound in $\Sigma^{1}$. By the Zorn lemma a minimal element exists.

Some notations. Let $p \cdot x$ denote the scalar product of $p$ and $x$ in $R^{n}$. If $K \subset R^{n}$ and $p \in R^{n}$, then $K_{p}$ is the $p$-boundary of $K$; namely $K_{p}=\{y \in$ $K: p \cdot y \geq p \cdot z$ for all $z \in K\}$. For $K \subset R^{n}$ and $y \in R^{n}$, we write $y+K$ for $\{y+z: z \in K\}$. We fix $p \in R^{n}$. Note that the set function $p \cdot \mu$, defined by $(p \cdot \mu)(A)=p \cdot \mu(A)$, is a $\sigma$-additive signed measure. For $T \in \Sigma$ we denote by $T_{+}, T_{-}$, and $T_{0}$ the decomposition of $T$ into sets, such that $p \cdot \mu$ is nonnegative on subsets of $T_{+}$and nonpositive on subsets of $T_{-}$, and such that $|p \cdot \mu|$ vanishes on $T_{0}$ and $T_{0}$ is maximal in the sense that $|p \cdot \mu|(A)=0$ then $|\mu|\left(A \backslash T_{0}\right)=0$ (namely $|\mu|$ is absolutely continuous with respect to $p \cdot \mu$ on $T_{+} \cup T_{-}$). It is easy to construct this decomposition (e.g. if $f(w)$ is the RadonNikodym derivative of $\mu$ with respect to $|\mu|$, then $T_{+}=\{w \in T: p \cdot f(w)>0\}$, etc.).

Proposition. Let $T \in \Sigma$. Then $(\operatorname{ch} R(T))_{p}=\mu\left(T_{+}\right)+\operatorname{ch} R\left(T_{0}\right)$.

Proof. The inclusion $\mu\left(T_{+}\right)+\operatorname{ch} R\left(T_{0}\right)$ in the $p$-boundary of $\operatorname{ch} R(T)$ is trivial. To verify the other direction, let $y \in(\operatorname{ch} R(T))_{p}$; we have to show that $y \in$ $\mu\left(T_{+}\right)+\operatorname{ch} R\left(T_{0}\right)$. Since for bounded sets the closure operation and taking convex-hull operation commute, it is enough to verify the inclusion for $y$ in the closure of $R(T)$; namely when $y=\lim \mu\left(T_{j}\right)$ and $T_{j} \subset T$. We claim that for $|\mu|\left(T_{+} \backslash T_{j}\right)$ and $|\mu|\left(T_{-} \cap T_{j}\right)$, both converge to zero as $j \rightarrow \infty$. This follows immediately from the convergence of $p \cdot \mu\left(T_{j}\right)$ to $p \cdot y=\max \{p \cdot z: z \in \operatorname{ch} R(T)\}$, and the splitting of $T$ into the positive, negative, and neutral parts with respect to $p \cdot \mu$. Once the convergence to zero of $|\mu|\left(T_{-} \cap T_{j}\right)$ and $|\mu|\left(T_{+} \backslash T_{j}\right)$ is established, we notice that $y$ is also the limit of $\mu\left(T_{+}\right)+\mu\left(T_{0} \cap T_{j}\right)$. The latter sequence is in $\mu\left(T_{+}\right)+\operatorname{ch} R\left(T_{0}\right)$, and this is what we have to show. 
Proof of the equality $x=\mu(S)$.

Case 1. $x$ is in the relative interior of $\operatorname{ch} R(S)$. Since the latter contains the zero vector, it follows that $x$ would also be in $\operatorname{ch} R\left(S^{1}\right)$ if $|\mu|\left(S \backslash S^{1}\right)$ is small enough. Such an $S^{1}$ with $|\mu|\left(S \backslash S^{1}\right)>0$ is easily constructed by the lack of atoms of $|\mu|$. This contradicts the minimality of $S$; thus $x$ cannot be in the relative interior of $\operatorname{ch} R(S)$.

Case 2. $x$ is in the relative boundary of $\operatorname{ch} R(S)$. Then a $p \in R^{n}$ exists with $x \in(\operatorname{ch} R(S))_{p}$ and $p \cdot x>p \cdot y$ for some $y \in R(S)$. By the proposition, $x-$ $\mu\left(S_{+}\right) \in \operatorname{ch} R\left(S_{0}\right)$, where $S_{+}$and $S_{0}$ are defined with respect to $p$. Clearly $S_{0}$ is a minimal set with this property; otherwise minimality of $S$ is contradicted. The linear dimensionality of $\operatorname{ch} R\left(S_{0}\right)$ is smaller than that of $\operatorname{ch} R(S)$; thus an induction argument (or repeating the argument $n-1$ times) completes the proof.

\section{BIBLIOGRAPHY}

1. M. DeWilde, Sur un théoreme de Lyapounov, Bull. Soc. Roy. Sci. Liège 38 (1969), 96-100.

2. H. Halkin, Some further generalizations of a theorem of Lyapunov, Arch. Rational Mech. Anal. 17 (1964), 272-277.

3. P. R. Halmos, The range of a vector measure, Bull. Amer. Math. Soc. 54 (1948), 416-421.

4. S. Koshi, A remark on Lyapunov-Halmos-Blackwell convexity theorem, Math. J. Okayama Univ. 14 (1969), 29-33.

5. A. A. Liapounoff, Sur les fonctions-vecteurs complètment additives, Izv. Akad. Nauk SSSR 4 (1940), 465-478.

6. J. Lindenstrauss, A short proof of Liapounoff's convexity theorem, J. Math. Mech. 15 (1966), 971-972.

7. C. Olech, The range of an unbounded vector valued measure, Math. Systems Theory 2 (1968), 251-256.

8. J. A. Yorke, Another proof of Liapounov convexity theorem, SIAM J. Control. Optim. 9 (1971), 351-353.

Department of Theoretical Mathematics, Weizmann Institute of Science, Rehovot 76100, ISRAEL 